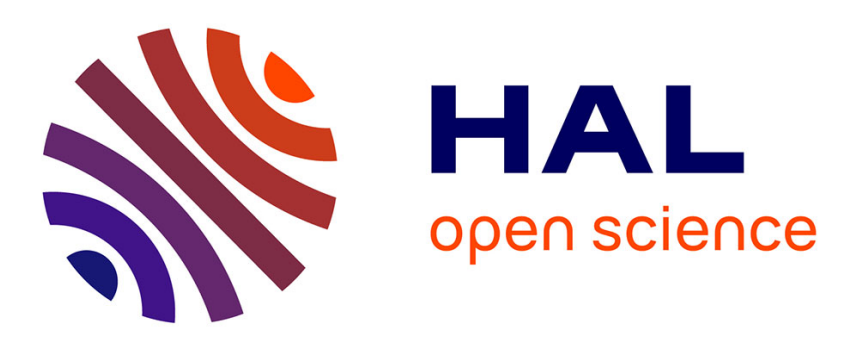

\title{
Direct estimation of generalized stress intensity factors using a three-scale concurrent multigrid X-FEM
} Jean-Charles Passieux, Anthony Gravouil, Julien Réthoré, Marie-Christine Baietto

\section{- To cite this version:}

Jean-Charles Passieux, Anthony Gravouil, Julien Réthoré, Marie-Christine Baietto. Direct estimation of generalized stress intensity factors using a three-scale concurrent multigrid X-FEM. International Journal for Numerical Methods in Engineering, 2011, 85 (13), pp.1648-1666. 10.1002/nme.3037 . hal-00708392

\section{HAL Id: hal-00708392 \\ https://hal.science/hal-00708392}

Submitted on 15 Jun 2012

HAL is a multi-disciplinary open access archive for the deposit and dissemination of scientific research documents, whether they are published or not. The documents may come from teaching and research institutions in France or abroad, or from public or private research centers.
L'archive ouverte pluridisciplinaire HAL, est destinée au dépôt et à la diffusion de documents scientifiques de niveau recherche, publiés ou non, émanant des établissements d'enseignement et de recherche français ou étrangers, des laboratoires publics ou privés. 


\title{
Direct estimation of generalized stress intensity factors using a three-scale concurrent multigrid X-FEM
}

\author{
J. C. Passieux ${ }^{1}$, A. Gravouil, J. Réthoré and M.C. Baietto \\ Laboratoire de Mécanique de Contacts et des Structures (LaMCoS), INSA de Lyon/CNRS \\ UMR5259/Université de Lyon, 20, avenue des Sciences, F-69621 Villeurbanne Cedex, France
}

\begin{abstract}
A concurrent multigrid method is devised for the direct estimation of stress intensity factors (SIF) and higher order coefficients of the elastic crack tip asymptotic field. The proposed method bridges three characteristic length scales that can be present in fracture mechanics: the structure, the crack and the singularity at the crack tip. For each of them, a relevant model is proposed. First, a truncated analytical reduced order model based on the Williams' expansion is used to describe the singularity at the tip. Then, it is coupled with a standard X-FEM model which is known to be suitable for the scale of the crack. A multigrid solver finally bridges the scale of the crack to that of the structure for which a standard FE model is often accurate enough. Dedicated coupling algorithms are presented and the effects of their parameters are discussed. The efficiency and accuracy of this new approach is exemplifyed using three benchmarks.
\end{abstract}

\section{Introduction}

The eXtended Finite Element Method (X-FEM [23, 24, 11]), was initially developed for the simulation of failure in solids. It is able to account for the displacement discontinuity across the crack and the asymptotic displacement at the crack tip by the introduction of additional enrichment functions into the classical finite element space, thanks to the Partition of the Unity (PUM) [22]. Its main advantage is that it allows the use of a mesh not matching the crack geometry. This avoids the re-meshing and, in the context of Linear Elastic Fracture Mechanics (LEFM), interpolation operations which can cause numerical errors and even possible numerical instabilities, when the crack propagates [29]. Unfortunately, the classical enrichment fields are not able to provide reliable estimates of the stress intensity factors (SIF) directly [7]. Generalized finite element and experimental methods [28] require post-processing to recover SIF, either with least-square methods, energy related techniques like interaction integrals or configuration forces (see for instance $[26,19]$ ).

Alternative tip enrichment techniques have been devised to simulate failure and yet allow for direct estimation of SIF. In this respect, Karihaloo \& Xiao [20] introduced a method which is still relying on the PUM, but with specific enrichment functions that are the Williams'

\footnotetext{
${ }^{1}$ Correspondance: E-mail: jean-charles.passieux@insa-lyon.fr Grant: Agence Nationale de la Recherche RUPXCUBE (ANR-09-BLAN-0009-01)
} 
series [35]. The area in which the nodes are enriched by the singular functions can be different from the area used to extract the SIFs (area where the degrees of freedom (DOF) associated with the same singular enrichment function are constrained to be equal) [20]. In [37], the underlying partition of the unity is inactivated by simply setting the regular DOFs to zero in the extraction zone. It was shown that these approaches do not predict accurately the coefficients of the higher order terms in the elastic crack tip asymptotic fields [37].

Another tip enrichment technique, no longer based on the PUM, called the Hybrid Crack Element (HCE) was initially proposed by Tong et al. [33] and extended to the determination of the coefficients of the higher order terms of the Williams' expansion in [15]. This last method provides more accurate SIF and also yields higher order coefficients precisely. Recently, a Hybrid Analytical and eXentended Finite Element Method (HAX-FEM) was proposed by Réthoré et al. [31]. This convenient and accurate tool couples two different models: an analytical reduced order model based on a truncated Williams' expansion in the vicinity of the crack tip and a standard X-FEM model with the discontinuous function along the crack faces alone. The models are coupled by partitioning the energy on an overlapping zone (Arlequin method [3]).

In the present paper, we propose a simpler version of HAX-FEM while providing the same level of accuracy. The analytical and the FE descriptions are no longer overlapping and their coupling is thus guaranteed by an integral matching technique (Mortar [4, 2]) on their intersection which restricts to an interface. This method is easier to implement, since no overlap is involved, and easier to use since the choice of two parameters (size of the overlap, energy weighting function) are avoided. The major advantage of this formulation is that it allows an easy coupling with a multigrid method as it is done afterwards. By the way, if this approach presents some similarities with HCE, the analytical model has the advantage to include directly the rigid body translations and rotation in the formulation whereas they need a special treatment to be recovered a posteriori with HCE [38].

All the methods listed before rely on the Williams' expansion which is dedicated to straight cracks only. In the general case, the singular enrichment zone must be defined on the scale on which the crack can be considered straight. Therefore the FE mesh must be fine enough to fit with this scale. Despite the difficulty of local refinement, the resulting mesh will lead to a larger system, whose resolution cost can become prohibitive for some complex problems. Dedicated strategies have been developed to handle these very different scales that are required to simulate cracked bodies. These methods can use energy coupling methods [3, 13], domain decomposition methods [12, 16], homogenization $[8,10,1]$ or generalized FEM [17]. Among them a multiscale method has been recently proposed and adapted to X-FEM [27] which is based on a multigrid solver $[5,25]$. In this paper we propose similarly a concurrent multigrid method inspired from HAX-FEM. It will be referred to as DEK-FEM for Direct Estimation of Generalized SIF $\left(K_{i}^{n}\right)$. A hierarchy of grids with increasing fineness and decreasing size is used to solve the problem. The solution is the substitution of the solutions of finer grids in coarser ones. The last and finest grid is the analytical model expressed in the Williams' formalism, whereas the remaining grids are formulated with classical X-FEM with jump and singular functions. An iterative scheme is proposed to couple these different scales.

The paper is organized as follows. After having recalled the properties of the elastic crack tip asymptotic fields in section 2, the proposed non-overlapping variant of HAX-FEM is presented in section 3. In section 4, the performances of the method are presented using two classical test cases. Section 5 summarizes the principle of local multigrid solvers and presents the proposed 
$D E K-F E M$ whose performances are illustrated in section 6 .

\section{Elastic crack tip asymptotic fields}

Let us consider a 2D isotropic elastic structure with an initial crack. The local coordinates centered at the crack tip are designated $r$ and $\theta$. If the crack is straight and has traction-free faces, the Williams' expansion of the displacement $\mathbf{u}(r, \theta)$ and stress field $\boldsymbol{\sigma}(r, \theta)$ are expressed by the following double series [35]:

$$
\begin{array}{r}
\mathbf{u}(r, \theta) \equiv\left[\begin{array}{c}
u_{x} \\
u_{y}
\end{array}\right]=\sum_{i=I, I I} \sum_{n=0}^{\infty} a_{i}^{n} \Phi_{i}^{n}(r, \theta) \\
\boldsymbol{\sigma}(r, \theta) \equiv\left[\begin{array}{cc}
\sigma_{x x} & \sigma_{x y} \\
(\text { sym }) & \sigma_{y y}
\end{array}\right]=\sum_{i=I, I I} \sum_{n=1}^{\infty} a_{i}^{n} \Psi_{i}^{n}(r, \theta)
\end{array}
$$

where $a_{i}^{n}$ are the coefficients of the linear elastic crack tip asymptotic fields and $\Phi_{i}^{n}$ and $\Psi_{i}^{n}$ the following reference fields:

$$
\begin{aligned}
& \Phi_{I}^{n}(r, \theta)=r^{n / 2} \quad\left[\begin{array}{c}
\left(\kappa+\frac{n}{2}+(-1)^{n}\right) \cos \frac{n}{2} \theta-\frac{n}{2} \cos \left(\frac{n}{2}-2\right) \theta \\
\left(\kappa-\frac{n}{2}-(-1)^{n}\right) \sin \frac{n}{2} \theta+\frac{n}{2} \sin \left(\frac{n}{2}-2\right) \theta
\end{array}\right] \\
& \Phi_{I I}^{n}(r, \theta)=\quad r^{n / 2} \quad\left[\begin{array}{l}
-\left(\kappa+\frac{n}{2}-(-1)^{n}\right) \sin \frac{n}{2} \theta+\frac{n}{2} \sin \left(\frac{n}{2}-2\right) \theta \\
\left(\kappa-\frac{n}{2}+(-1)^{n}\right) \cos \frac{n}{2} \theta+\frac{n}{2} \cos \left(\frac{n}{2}-2\right) \theta
\end{array}\right] \\
& \Psi_{I}^{n}(r, \theta)=\frac{n}{2} r^{n / 2-1}\left[\begin{array}{cc}
\left(2+\frac{n}{2}+(-1)^{n}\right) \cos \left(\frac{n}{2}-1\right) \theta & \left(\frac{n}{2}-1\right) \sin \left(\frac{n}{2}-3\right) \theta-\ldots \\
\ldots-\left(\frac{n}{2}-1\right) \cos \left(\frac{n}{2}-3\right) \theta & \left(\frac{n}{2}+(-1)^{n}\right) \sin \left(\frac{n}{2}-1\right) \theta \\
(\operatorname{sym}) & \left(2-\frac{n}{2}-(-1)^{n}\right) \cos \left(\frac{n}{2}-1\right) \theta \\
\ldots+\left(\frac{n}{2}-1\right) \cos \left(\frac{n}{2}-3\right) \theta
\end{array}\right] \\
& \Psi_{I I}^{n}(r, \theta)=\frac{n}{2} r^{n / 2-1}\left[\begin{array}{cc}
\left(\frac{n}{2}-1\right) \sin \left(\frac{n}{2}-3\right) \theta-\ldots & \left(\frac{n}{2}-1\right) \cos \left(\frac{n}{2}-3\right) \theta-\ldots \\
\left(2+\frac{n}{2}-(-1)^{n}\right) \sin \left(\frac{n}{2}-1\right) \theta & \left(\frac{n}{2}-(-1)^{n}\right) \cos \left(\frac{n}{2}-1\right) \theta \\
(\operatorname{sym}) & \left(\frac{n}{2}-2-(-1)^{n}\right) \sin \left(\frac{n}{2}-1\right) \theta \\
\ldots-\left(\frac{n}{2}-1\right) \sin \left(\frac{n}{2}-3\right) \theta
\end{array}\right]
\end{aligned}
$$

where $\kappa$ is the Kolossov constant such that $\kappa=3-4 \nu$ for plane strain or $\kappa=((3-\nu) /(1+\nu))$ for plane stress conditions; $\nu$ being Poisson's ratio.

Let us recall some notable properties of this expansion. First, $a_{I}^{0}, a_{I I}^{0}$ and $a_{I I}^{2}$ are the rigid body translations and rotation with respect to the crack tip, respectively. Second, $a_{I}^{2}$ is the so called $T$-stress component for mode $I$. And finally, first order $(n=1)$ coefficients $a_{I}^{1}$ and $a_{I I}^{1}$ are related to the mode I and II stress intensity factors (SIFs) as:

$$
a_{I}^{1}=\frac{K_{I}}{2 \mu \sqrt{2 \pi}} \quad \text { and } \quad a_{I I}^{1}=\frac{K_{I I}}{2 \mu \sqrt{2 \pi}}
$$

where $\mu$ is the second Lamé's coefficient. Each odd term $\Phi_{i}^{2 p+1}$ is discontinuous across the crack mouth and each even term $\Phi_{i}^{2 p}$ is continuous. 


\section{Monoscale formulation}

The domain is divided into two non-overlapping sub-domains $\Omega_{X}$ and $\Omega_{W}$, see Figure 1 , respectively characterized by the restriction $u_{X}$ and $u_{W}$ of the unknown displacement. Subdomain $\Omega_{W}$ is viewed as a patch which incorporates the crack tip whereas $\Omega_{X}$ is the outer domain which holds the boundary conditions, i.e. traction forces $F_{d}$ on part $\partial_{2} \Omega_{X}$ of the boundary and prescribed displacement $u_{d}$ on its complementary $\partial_{1} \Omega_{X}$. Let us introduce

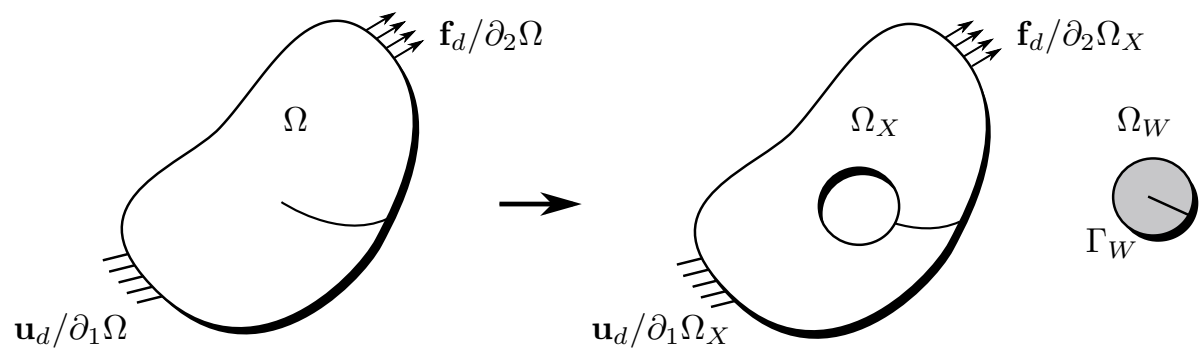

Figure 1: decomposition of the domain into an X-FEM domain and an analytical patch

$\mathcal{U}_{X}=\left\{\mathbf{u}_{X} \in H^{1}\left(\Omega_{X}\right) \mid \mathbf{u}_{X}=\mathbf{u}_{d}\right.$ on $\left.\partial_{1} \Omega_{X}\right\}$, the space of admissible displacements and $\mathcal{U}_{X}^{0}$ its corresponding vector space. Spaces $\mathcal{U}_{W}$ and $\mathcal{U}_{W}^{0}$ are defined in the same way - one can notice that $\mathcal{U}_{W}=\mathcal{U}_{W}^{0}$ since the crack faces are assumed to be traction-free- . On each domain $\Omega_{i}$ $(i=X, W)$ the mechanical problem reads: find $\mathbf{u}_{i} \in \mathcal{U}_{i}$, such that:

$$
\begin{gathered}
a_{i}\left(\mathbf{u}_{i}, \mathbf{u}_{i}^{\star}\right)=l_{i}\left(\mathbf{u}_{i}^{\star}\right) \quad \forall \mathbf{u}_{i}^{\star} \in \mathcal{U}_{i}^{0} \quad \forall i=X, W \\
\text { with } \quad a_{i}\left(\mathbf{u}_{i}, \mathbf{u}_{i}^{\star}\right)=\int_{\Omega_{i}} \varepsilon\left(\mathbf{u}_{i}^{\star}\right): \mathbb{C} \varepsilon\left(\mathbf{u}_{i}\right) d \Omega \quad \text { and } \quad l_{X}\left(\mathbf{u}_{X}^{\star}\right)=\int_{\partial_{2} \Omega} \mathbf{u}_{X}^{\star} \mathbf{f}_{d} d s
\end{gathered}
$$

where $\mathbb{C}$ is the Hooke tensor. Note that $l_{W}$ is zero since the crack faces are traction free.

In order to close the problem, one needs to impose the compatibility of displacement fields. In opposition with the method proposed in [31], the sub-domains are non-overlapping, their intersection reduces to an interface denoted $\Gamma_{W}$. Thus the coupling is no longer ensured thanks to a partition of the energy technique (i.e. the Arlequin method [3]). In the method proposed herein, the coupling is provided by integral matching, like in [18]. The displacement continuity condition on $\Gamma_{W}$ is enforced in a weak sense, by introducing a Lagrange multiplier $\lambda \in \mathcal{L}$ (where $\mathcal{L}$ is the ad-hoc space whose discretization is discussed in section 6) and the following scalar product:

$$
\left\langle\mathbf{u}_{X}-\mathbf{u}_{W}, \boldsymbol{\lambda}\right\rangle_{\Gamma}=\int_{\Gamma_{W}} \boldsymbol{\lambda}\left(\mathbf{u}_{X}-\mathbf{u}_{W}\right) d s=0
$$

The entire three field formulation reads: find $\left(\mathbf{u}_{X}, \mathbf{u}_{W}, \boldsymbol{\lambda}\right) \in \mathcal{U}_{X} \times \mathcal{U}_{W} \times \mathcal{L}$, such that:

$$
a_{X}\left(\mathbf{u}_{X}, \mathbf{u}_{X}^{\star}\right)+a_{W}\left(\mathbf{u}_{W}, \mathbf{u}_{W}^{\star}\right)+\left\langle\mathbf{u}_{X}^{\star}-\mathbf{u}_{W}^{\star}, \boldsymbol{\lambda}\right\rangle_{\Gamma}+\left\langle\mathbf{u}_{X}-\mathbf{u}_{W}, \boldsymbol{\lambda}^{\star}\right\rangle_{\Gamma}=l_{X}\left(\mathbf{u}_{X}^{\star}\right)
$$

Subdomains $\Omega_{X}$ and $\Omega_{W}$ differ in the description of the displacement fields. Here the same interpolation as HAX-FEM [31] is used. In the sequel, a brief review of the main aspects is given. 


\subsection{X-FEM domain}

On subdomain $\Omega_{X}$ a classical X-FEM is used. It relies on a finite element mesh where $N$ is the set of nodes and $\left\{\phi_{i}\right\}_{i \in N}$ the finite element shape functions defined on their support. Here, the region near the crack tip being in the complementary sub-domain $\Omega_{W}$, the discontinuous enrichment alone is required. It allows for taking into account the displacement jump across the crack, for the nodes $N_{c} \in N$ whose support is cut. In this domain, the displacement interpolation reads:

$$
\mathbf{u}_{X}(x)=\sum_{i \in N} \phi_{i}(x) q_{i}+\sum_{i \in N_{c}} \phi_{i}(x) H(x) b_{i}=\mathbf{N}_{X}^{T} \mathbf{U}_{X}
$$

Where $q_{i}$ are the regular DOFs. $H$ is the symmetric Heaviside enrichment function and $b_{i}$ its corresponding DOFs. $\mathbf{N}_{X}$ denotes the matrix which collects the values of the interpolation functions and $\mathbf{U}_{X}$ its corresponding DOF vector.

\subsection{Analytical patch}

On sub-domain $\Omega_{W}$, no finite element mesh is involved. In fact, the interpolation of the displacement is only defined by a truncated Williams' expansion (1):

$$
\mathbf{u}_{W}(x)=\sum_{i=I, I I} \sum_{n=0}^{n_{\max }} a_{i}^{n} \Phi_{i}^{n}(r, \theta)=\mathbf{N}_{W}^{T} \mathbf{U}_{W}
$$

where $\mathbf{N}_{W}$ denotes the matrix which collects the values of the interpolation functions and $\mathbf{U}_{W}$ its corresponding DOF vector. Note that the SIFs and higher order terms are special DOFs of the model and can thus be directly extracted from $\mathbf{U}_{W}$ thanks to proportionality relations, like $(3)$.

\subsection{Matching interface}

The Lagrange multiplier $\boldsymbol{\lambda}$ can be discretized either with extended finite elements or with analytical interpolation functions based on the Williams' expansion. The interpolation of the Lagrange multiplier can be written in the general form:

$$
\boldsymbol{\lambda}(x)=\sum_{i} \phi_{i}(x) l_{i}=\mathbf{N}_{L}^{T} \boldsymbol{\Lambda}
$$

where again $\mathbf{N}_{L}$ denotes the matrix which collects the values of the interpolation functions and $\boldsymbol{\Lambda}$ the corresponding DOF vector.

\subsection{Practical implementation}

In practice, Domain $\Omega$ is first meshed with standard linear finite element (Tri3 or Quad4). Then, the elements of $\Omega_{X}$ near the crack tip are simply removed from the X-FEM sub-domain and used to generate the integration support for the analytical patch. The interface $\Gamma_{W}$ is then directly endowed with a mesh which is the inner skin mesh of the X-FEM domain. This is convenient for the computation of the matching operators. Standard extended finite element 
shape functions on the skin elements will be used, in the examples below. This point is further discussed in Section 5.

For integration, the elements cut by the crack are subdivided into a set of elements whose boundaries align with the crack geometry, following [23]. Then, standard second order Gauss Legendre quadrature is used in sub-domain $\Omega_{X}$. However, since discontinuous enrichments alone are involved, one could have used standard quadrature without splitting the elements cut, by using an apropriate technique [34]. To ensure that the strain field is adequately integrated, especially for the singular terms $(\mathrm{n}=1)$, in the analytical patch $\Omega_{W}$, higher order Gauss quadrature is required. In the examples below, fifth order is used (25 Gauss nodes for Quad4 and 7 Gauss nodes for Tri3). According to our experiments, the effect of higher orders of quadrature is rather slight on the accuracy of SIFs.

With such a discretization of (6), one can obtain the following algebraic problem: find $\mathbf{U}_{X}$, $\mathbf{U}_{W}, \boldsymbol{\Lambda}$, such that:

$$
\left[\begin{array}{ccc}
\mathbf{K}_{X} & 0 & \mathbf{C}_{X}^{T} \\
0 & \mathbf{K}_{W} & -\mathbf{C}_{W}^{T} \\
\mathbf{C}_{X} & -\mathbf{C}_{W} & 0
\end{array}\right]\left[\begin{array}{c}
\mathbf{U}_{X} \\
\mathbf{U}_{W} \\
\boldsymbol{\Lambda}
\end{array}\right]=\left[\begin{array}{c}
\mathbf{F}_{d} \\
0 \\
0
\end{array}\right]
$$

where $\mathbf{K}_{X}$ (resp. $\mathbf{K}_{W}$ ) is the stiffness matrix of subdomain $\Omega_{X}$ (resp. $\Omega_{W}$ ). $\mathbf{F}_{d}$ is the generalized force vector and $\mathbf{C}_{i}$ denoted the integral of the cross product of the non conforming shape functions $\mathbf{N}_{L}$ and $\mathbf{N}_{i}$, which are known as the mortar projector [4, 2]; they are defined by:

$$
\begin{aligned}
\mathbf{K}_{i} & =\int_{\Omega_{i}} \varepsilon\left(\mathbf{N}_{i}^{T}\right): \mathbb{C} \varepsilon\left(\mathbf{N}_{i}\right) d \Omega \quad \forall i=X, W \\
\mathbf{C}_{i} & =\int_{\Gamma_{W}} \mathbf{N}_{i}^{T} \mathbf{N}_{L} d s \quad \forall i=X, W \\
\mathbf{F}_{d} & =\int_{\partial_{2} \Omega_{X}} \mathbf{N}_{X}^{T} \mathbf{f}_{d} d s
\end{aligned}
$$

With such a formulation, the method presents some similarities with the Hybrid Crack Element (HCE) proposed in [15]. However, besides the fact that here, no special mesh is required a priori, the rigid body modes that are excluded in the formulation of the HCE, need a least square treatment to be recovered a posteriori, in order to determine the complete displacement field [38], which is not the case in the proposed approach. This is made possible at the expense of integrals in the volume of the analytical model.

\section{$4 \quad$ Numerical examples}

Two classical benchmarks are studied to demonstrate the efficiency of the proposed monoscale method to estimate directly SIFs and higher order terms. First, an edge-cracked plate in remote tension (ECPT) is used to compare the efficiency of the method with that of [31]. Then a wedge splitting specimen (WS), case for which an in-depth study is provided by [14], shows that the method is also appropriate for the accurate estimation of higher order terms of the Williams' expansion. This last example investigates also the case of unstructured meshes. 


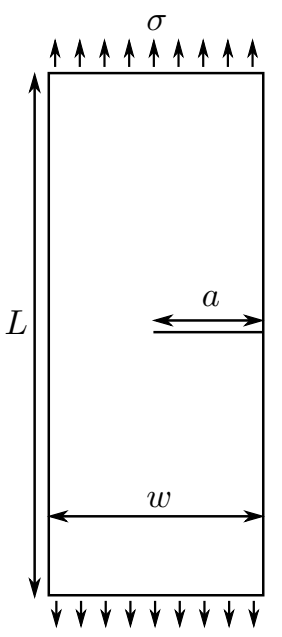

(a) geometry and loading

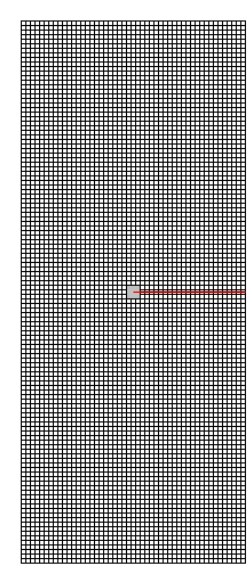

(b) mesh and patch for $r_{W}=2$

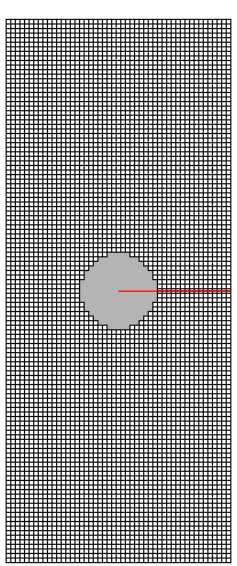

(c) mesh and patch for $r_{W}=8$

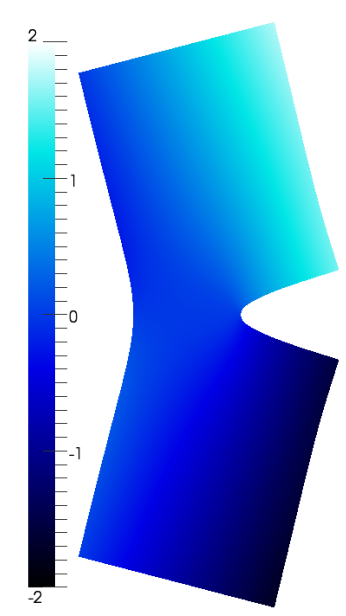

(d) vertical displacement in $\mu \mathrm{m}($ ampl. fact. $\times 1000)$

Figure 2: Edge cracked plate under remote tension

\subsection{Edge-cracked plate under remote tension}

Let us consider an edge-cracked plate, as shown in Figure 2(a), subjected to a uniform tensile stress $\sigma=10 \mathrm{MPa}$. The plate has a length $L=17 \mathrm{~mm}$, width $w=7 \mathrm{~mm}$ and the length of the crack is $a=3.5 \mathrm{~mm}$. The structure is considered to be in plane strain conditions, with a Poisson's ratio equal to $\nu=0.3$ and Young's modulus $E=200 \mathrm{GPa}$. The mesh of subdomain $\Omega_{X}$ is regular and made of $49 \times 119$ bilinear quadrangular elements with the aforementioned Gauss-Legendre quadrature scheme. The size of $\Omega_{W}$ is parametrized by a radius $R_{W}=h r_{W}$ ( $h$ being the average element size of the mesh of $\Omega_{X}$ ). The mesh, the patch and the crack are plotted on Figure 2(b) (resp. Figure 2(c)) for $r_{W}=2$ (resp. $r_{W}=8$ ). Figure 2(d) shows the vertical displacement field for $r_{W}=2$ in the deformed domain. For such a problem, the value of $K_{I}$ can be accurately approximated by the one that holds for an infinite plate, corrected by a factor depending on the ratio $a / w[6]$. In the sequel the value $K_{I 0}=2.9637 \mathrm{MPa} \sqrt{\mathrm{m}}$ is used as the reference.

For different values of the order of truncation $n_{\max }$ of the williams' expansion, the normalized value of $K_{I}$ is plotted as a function of the size of the analytical patch $r_{W}$ on Figure $3(\mathrm{a})$ for the Arlequin matching (from [31]) and on Figure 3(b) for the integral matching proposed herein. A comparable level of accuracy is obtained and the dependence on the size of the patch observed for $n_{\max }=2$ vanishes similarly when higher order terms are taken into account.

Remark. When $r_{W}=1$ the value of $K_{I}$ seems to be less accurate. This is because the patch is glued to a linear element whose kinematic description is too poor. This is not the case with the Arlequin matching since, even when $r_{\text {inner }}=1$, the patch overlaps a least 2 layers of nodes.

Again, for different values of $n_{\text {max }}$, the effect of the mesh size $h$ on the accuracy of $K_{I}$ is shown Figure 4(a) for the Arlequin matching with $r_{\text {inner }}=1$ and $r_{\text {outer }}=2$ and Figure 4(b) for the integral matching with $r_{W}=2$. 


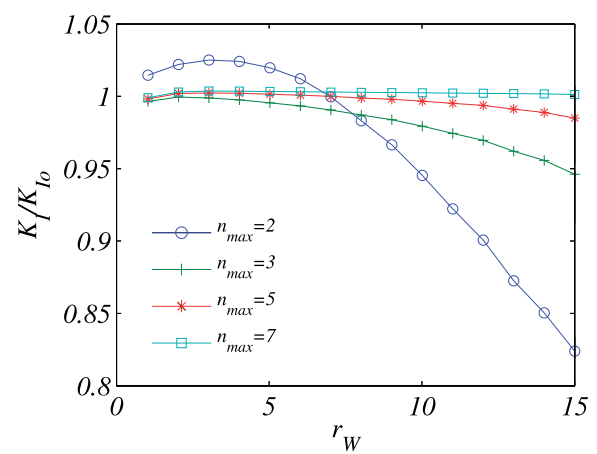

(a) Arlequin Matching with $l_{\text {overlap }}=1$

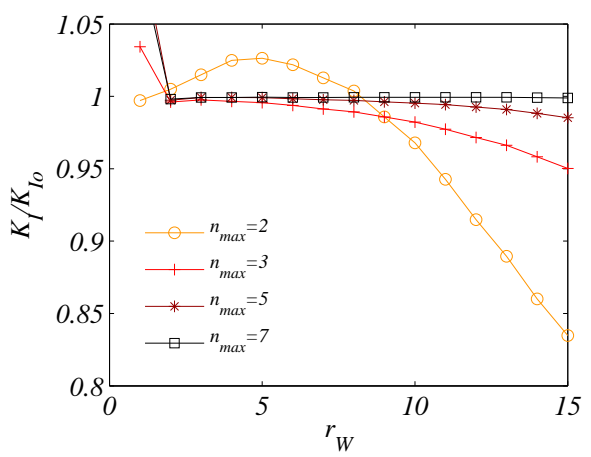

(b) Integral Matching

Figure 3: Normalized $K_{I}$ as function of the size of the patch for different values of $n_{\max }$.

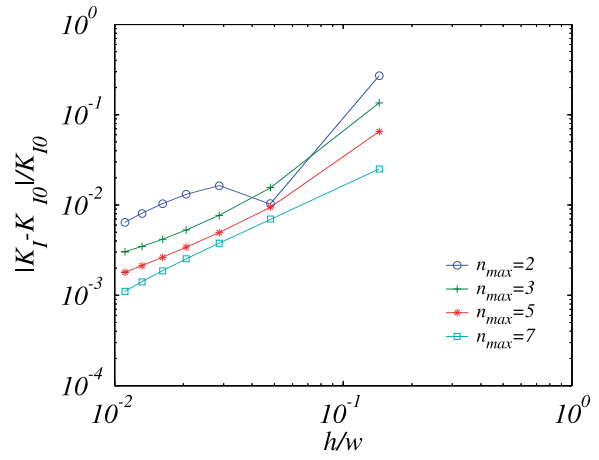

(a) Arlequin Matching with $r_{\text {inner }}=1$ and $r_{\text {outer }}=2$

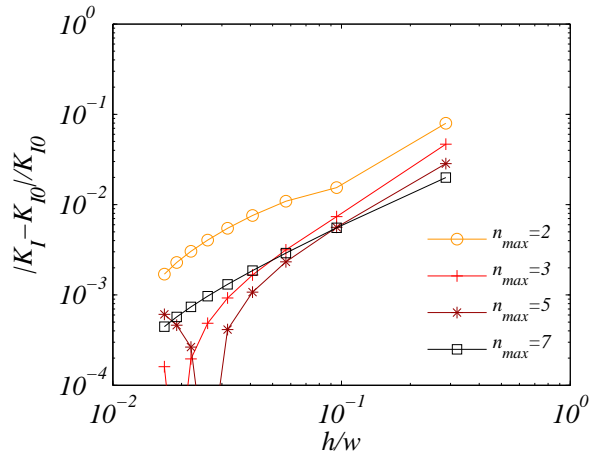

(b) Integral Matching with $r_{W}=2$

Figure 4: Relative error on $K_{I O}$ as function of the mesh size for different values of $n_{\max }$. 
For both methods, the convergence rates are between 1.5 and 1.25. With Arlequin, the matching is performed in the overlapping zone, namely between $r=1$ and $r=2$, whereas for integral matching $r=2$. It explains that the accuracy of the SIF on Figure 4(b) is slightly better than that of Figure 4(a).

This example shows that the proposed hybrid analytical and extended finite element with a non-overlapping integral matching is almost as efficient as the one with Arlequin matching initially proposed in [31] for the estimation of the stress intensity factor. Besides the fact that no overlap is involved, the advantage of integral matching is that the method is now more likely to fit into the framework of multigrid methods, as it is done in Section 5 of this paper.

\subsection{Wedge Splitting Specimen}

In the previous example, only the accuracy of the first order coefficient $n=1$ was discussed. In order to see the capabilities of this method to estimate the higher order coefficients $a^{n}$, we consider a wedge splitting specimen as shown in Figure 5(a). It consists of a square domain with a groove in which a splitting force is applied by the wedge. The force is assumed to be a distributed load $p=1 \mathrm{MPa}$ within the groove. The specimen is supported on a two line support. The crack is localized at the bottom of the groove and its length $c$ varies from 4 to $64 \mathrm{~mm}$. The Young's modulus $E$ is set at $100 \mathrm{GPa}$ and Poisson's ratio $\nu$ at 0.25 . A state of plane stress is considered.

The domain is first meshed by an unstructured grid with linear Tri3. The reduced order model expressed by the Williams' expansion $\Omega_{W}$ is defined in the region occupied by $r_{W}=3$ layers of elements around the crack, the remainder defining $\Omega_{X}$. This modeling and its solution is shown in Figure 5(b), 5(c) and 5(d) for a crack of length $c=4 \mathrm{~mm}, c=40 \mathrm{~mm}$ and $c=64 \mathrm{~mm}$, respectively. The analytical patch is in grey and the Von-Mises stress field is plotted on the deformed structure. Note that one unique and rather coarse mesh was used for the different crack lenghts.

In [14], this problem was solved with a high order HCE (up to 39 terms of the Williams' expansion) together with a relatively fine structured discretization of the remainder of the body. Accurate analytical expression were obtained by fitting the computed SIF, T-stress and the third to fifth coefficients. For different values of the crack length $c$, the problem is now solved with the proposed method for $n_{\max }=9$. The computed normalized coefficients $\left\{a_{I}^{n}(c) / a_{I}^{n}(64 \mathrm{~mm})\right\}_{1 \leq n \leq 5}$ are plotted on Figure 6 and compared to those obtained with HCE as well as their polynomial extrapolation [14]. One can observe an excellent agreement $(\approx 1 \%)$ between the results obtained with high order HCE and those computed here with a unique and rather coarse unstructured mesh.

This example shows that, besides the good estimation of the stress intensity factors, the proposed technique also yields accurate high order terms of the crack tip asymptotic fields. In addition, this technique seems well suited for crack propagation without remeshing and its robustness allows us to consider arbitrary meshes.

The aim of the first part of this work was to propose a simplified alternative method, making sure it is as accurate as HAX-FEM. We showed that the non-overlapping approach actually has the same efficiency that the original HAX-FEM. The method is simpler to implement, since there is no overlap. Moreover, the choice of two parameters related to the overlap are avoided (the size of the overlap, and the energy weighting function) which makes it easier to use. In the following section, we will see that, in addition, the proposed formulation is more 


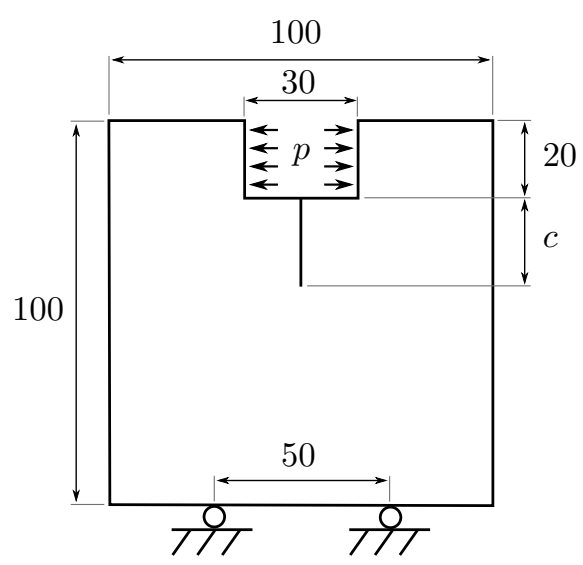

(a) geometry $(\mathrm{mm})$ and loading

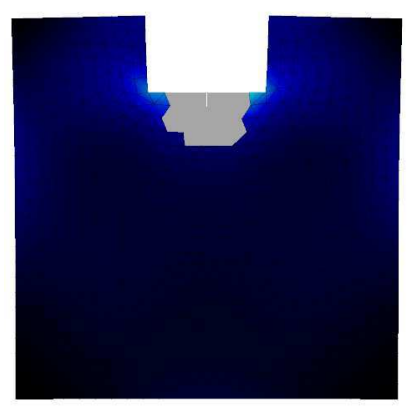

(b) mesh and patch for $c=$ $4 \mathrm{~mm}$

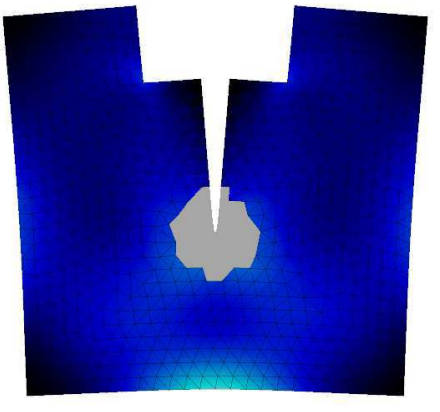

(c) mesh and patch for $c=40 \mathrm{~mm}$

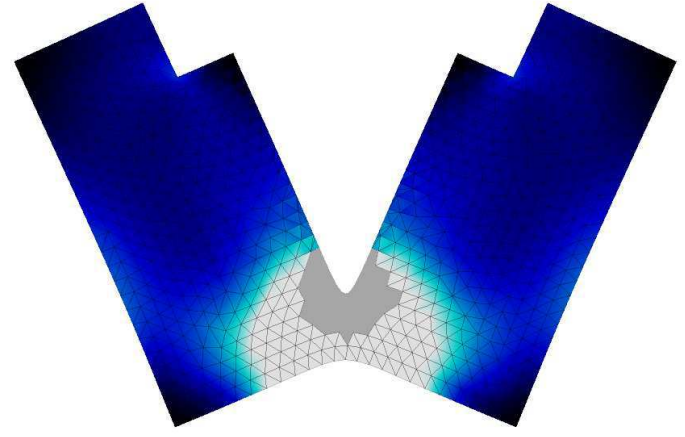

(d) mesh and patch for $c=64 \mathrm{~mm}$
4

3

2

1

0

(e)

Figure 5: Wedge splitting specimen with distributed load and two line supports. (a) Problem definition, (b,c,d) Von Mises Stress solution (in MPa) for different crack length and (e) the common colorbar. (ampl. fact. $\times 1000$ )

likely to be integrated in a multigrid framework.

\section{Multiscale formulation}

The efficiency of this method rests on the Williams' expansion which is only valid if the crack is straight, at least in the analytical patch. Therefore, the initial mesh needs to be locally refined, enough to legitimate this assumption. Besides, even with a straight crack, the FE convergence study of the previous section shows that the mesh needs to be refined if one wants to get an accurate determination of the SIF. In this case, it was shown in [27] that a localized refinement is enough. Despite the fact that a refinement step can be difficult and time consuming, it leads to a larger system whose resolution cost can become prohibitive for some complex problems and especially for 3D problems. In the sequel, we devise a concurrent multigrid method, inspired from [27], able to bridge the scale of the structure to that of the 


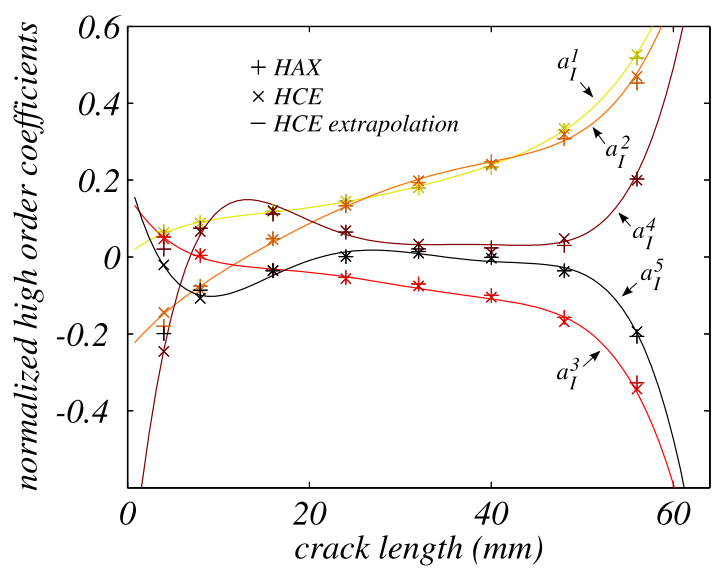

Figure 6: Accuracy of the computed high order terms $a_{I}^{n} / a_{I}^{n}(64 m m)$

singularity at the crack tip and to yield generalized SIF such as the method of the previous section.

The basic idea of multigrid methods $[5,25]$ is that for iterative solvers such as Jacobi, GaussSeidel or some Krylov solvers, the high frequency part of the solution is accurately computed after few iterations on a fine grid, whereas it would take a large number of iterations to compute the remaining smooth part of the solution properly on this given grid. This is called the error smoothing property. To avoid this drawback, multigrid methods involve resolutions on a hierarchy of grids with a decreasing fineness with possible different sizes (if localized). Therefore, the smoothed residual is transferred (restriction operation) to the coarser grids which are able to compute rapidly the low frequency part of the solution. This process is completed until the mesh is coarse enough to use a direct solver. Then, the solution on the finer grids are successively improved with the smooth corrections (prolongation operation), in order to accelerate the convergence of the iterative algorithm on the finest grid. This scheme, called the multigrid cycle, is completed once per iteration until convergence. A prolongation operator $\mathbf{P}$ (resp. restriction $\mathbf{R}$ ) is introduced to transfer the displacement (resp. residual) from the coarse mesh to the fine (resp. fine to coarse). Their definition allows us to handle naturally the mesh incompatibilities.
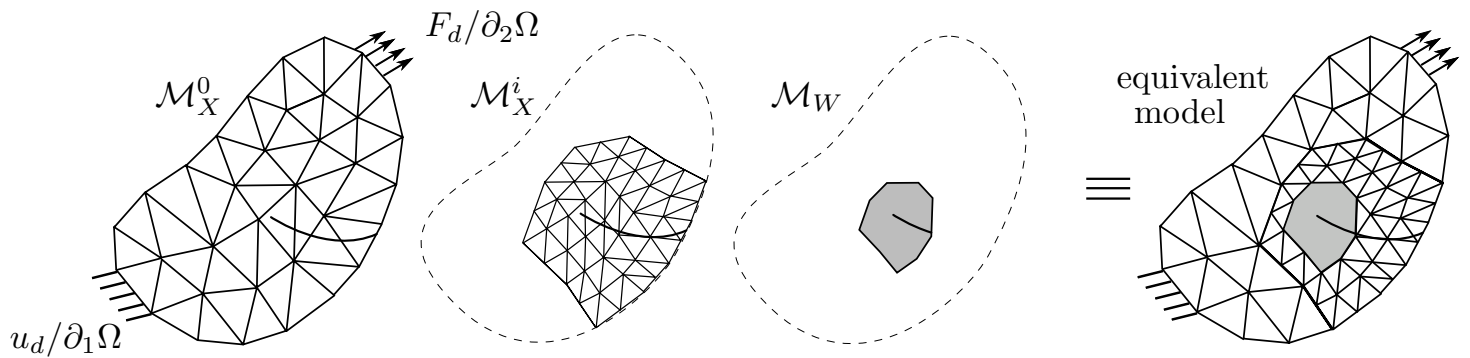

Figure 7: $D E K-F E M$ : hierarchy of X-FEM grids $\mathcal{M}_{X}^{i}$ and analytical model $\mathcal{M}_{W}$. The equivalent model corresponds to the substitution of the finers models in the coarser one. 
In the following, the domain $\Omega$ is endowed with a series of localized meshes of different grid sizes (Figure 7). For this, an initial coarse mesh $\mathcal{M}_{X}^{0}$ of the whole domain is set up and then, localized meshes $\mathcal{M}_{X}^{i}$ including the crack tip are recursively generated. Here, the refinement is performed by splitting the elements, so that the grids are nested. The ratio of fineness between two consecutive grids is set to 2 which seems to be optimal [21]. Once again, three kinds of models are involved. The last and smaller grid is the integration support of the analytical reduced order model expressed in the Williams' formalism $\mathcal{M}_{W}$. On the remaining grids $\mathcal{M}_{X}^{i}$, a full X-FEM model with jump and classical singular enrichments [23] is used. Following [27], the grids whose elements are larger than the size of the crack use standard FEM only. After convergence, the solution of the multigrid algorithm corresponds to the substitution of the solution of the finer models in the solution of the coarser models, as shown in Figure 7.

The multigrid methods can differ in the definition of the intergrid operations depending on the models used on the different levels. Here, because of the two models, two different strategies are required to link one model to another. First, between two X-FEM grids $\mathcal{M}_{X}^{i}$, $\mathcal{M}_{X}^{i+1}$, the localized multigrid method dedicated to X-FEM (denoted LMG-X-FEM) proposed by Rannou et al. [27] will be used. It is briefly reviewed in Section 5.1. Secondly, a multigridlike algorithm is being devised between the last X-FEM grid and the analytical patch $\mathcal{M}_{W}$ in Section 5.2.

\subsection{Multigrid algorithm between X-FEM grids}

Between X-FEM grids, one uses the LMG-X-FEM which was recently proposed [27] to handle $\mathrm{X}$-FEM models in a localized multigrid framework. A brief review of the mains aspects of this method is given in this paragraph, for further details, see [27].

Subscript $\square_{X}$ is omitted in this section, since all the quantities refer to X-FEM models. For the sake of simplicity, let us consider a two-level LMG algorithm defined on the grids of Figure 8, made of one coarse mesh $\overline{\mathcal{M}}^{A B}$ and one fine mesh $\mathcal{M}^{B}$ localized around the crack. The
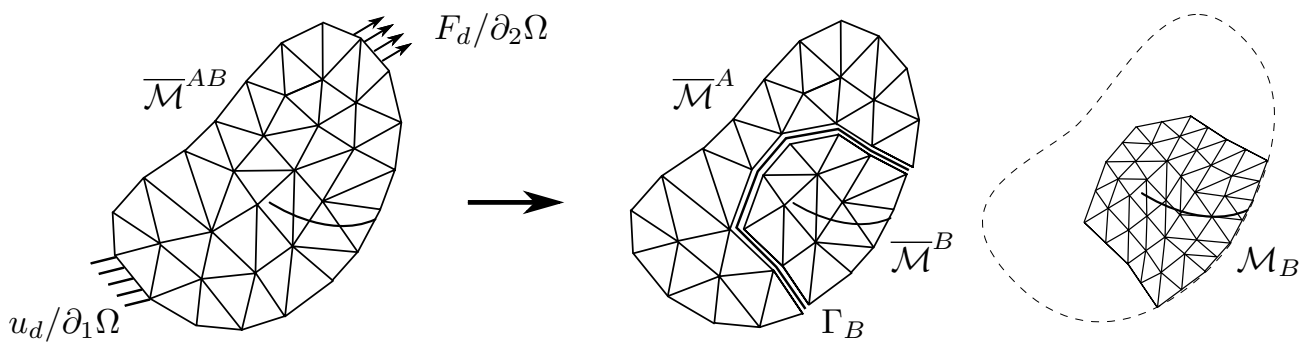

Figure 8: Grid hierarchy: Coarse mesh $\overline{\mathcal{M}}^{A B}$ and local fine mesh $\mathcal{M}^{B}$

coarse mesh is viewed as the sum of two sub-meshes $\overline{\mathcal{M}}^{B}$ the part of the mesh overlapped by the fine mesh and $\overline{\mathcal{M}}^{A}$ its complementary part. $\Gamma_{B}$ defines the interface between these two domains. In the sequel, the quantities $\bar{\square}^{A B}, \bar{\square}^{A}, \bar{\square}^{B}$ and $\square^{B}$ are respectively the discretization of $\square$ on the meshes $\overline{\mathcal{M}}^{A B}, \overline{\mathcal{M}}^{A}, \overline{\mathcal{M}}^{B}, \mathcal{M}^{B}$.

The prolongation $\mathbf{P}$ (and restriction $\mathbf{R}=\mathbf{P}^{T}$ ) operator is computed thanks to the collocation method given in [27] which is proved to be accurate enough. One iteration of LMG-X-FEM is given in Algorithm 1. It is made of three sub-stages: (1) the coarse problem whose right-hand- 


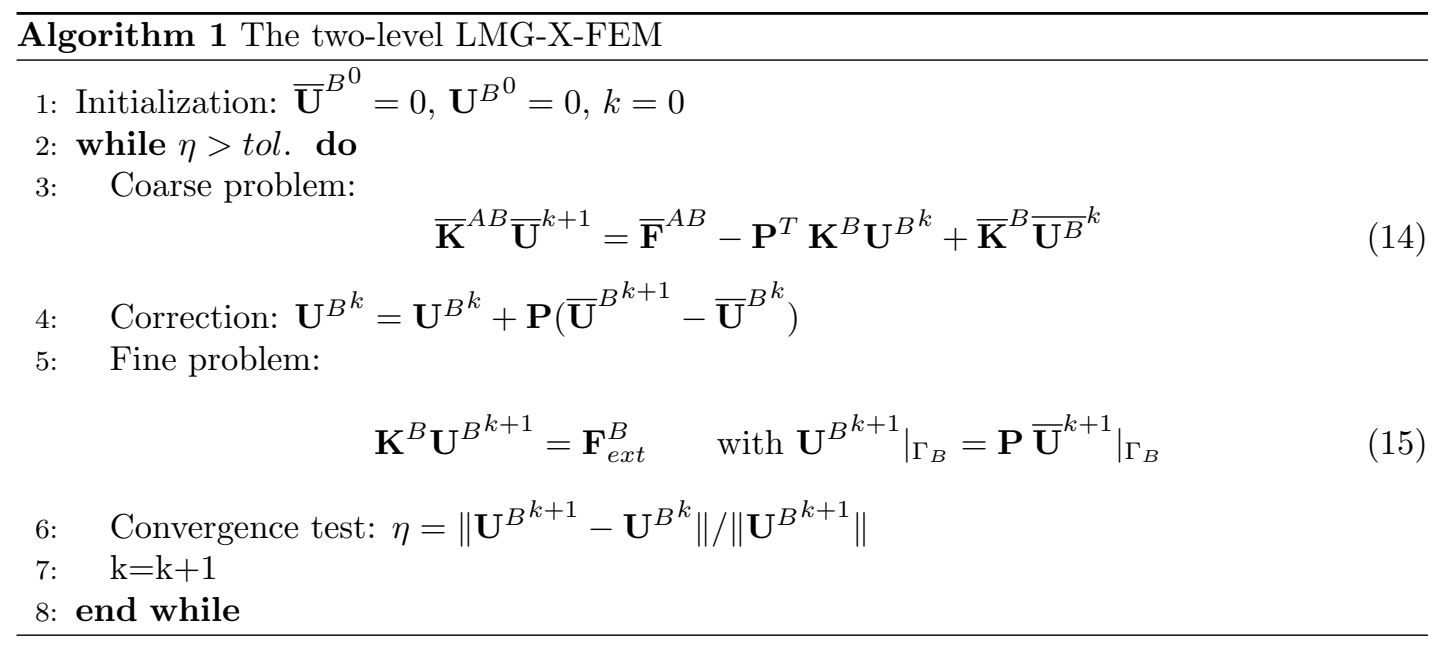

side involves the loading $\overline{\mathbf{F}}^{A B}$ and two additional terms whose role is to inactivate the coarse stiffness $\overline{\mathbf{K}}^{B} \overline{\mathbf{U}}^{B}$ and replace it by the restriction of the fine $\mathbf{K}^{B} \mathbf{U}^{B^{k}}$. This problem is solved until convergence and yields $\overline{\mathbf{U}}^{k+1}$. (2) The fine iterate $\mathbf{U}^{k}$ is improved by the prolongation of the coarse correction. (3) This corrected solution is then smoothed by few iterations (usually less than five) of the iterative solver on the fine problem defined on $\Omega_{W}$ with the coarse displacement $\overline{\mathbf{U}}^{A B}$ prescribed on its boundary $\Gamma_{B}$. In the examples below the solver used is a Conjugate Gradient possibly preconditioned by an incomplete Cholesky factorization.

Using a larger sequence of grids increases the efficiency of the multigrid algorithm. In this case, the coarse problem is no longer solved by a direct solver, but by a recursive call to the multigrid algorithm. The number of multigrid sub-cycles for each stage is taken to $\gamma_{X}=1$ for the X-FEM models.

\subsection{Multigrid sub-cycle between HAX-FEM and X-FEM}

Let us focus on the last X-FEM grid $\mathcal{M}_{X}^{A B}$ and the analytical patch $\mathcal{M}_{W}$ of Figure $9 . \mathcal{M}_{X}^{A}$
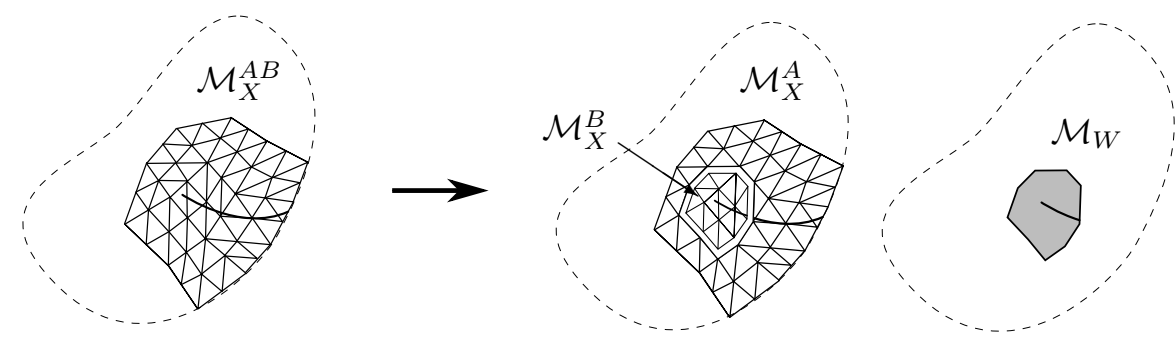

Figure 9: Grid hierarchy: Finest mesh $\overline{\mathcal{M}}_{A B}$ and analytical patch $\mathcal{M}_{W}$

defines the part of $\mathcal{M}_{X}^{A B}$ that is not overlapped by $\mathcal{M}_{W}$ and $\mathcal{M}_{X}^{B}$ the remainder. In this section, the quantities $\square_{X}^{A B}, \square_{X}^{A}, \square_{X}^{B}$ and $\square_{W}$ are respectively the discretization of $\square$ on the 
meshes $\mathcal{M}_{X}^{A B}, \mathcal{M}_{X}^{A}, \mathcal{M}_{X}^{B}, \mathcal{M}_{W}$. In opposition with Section 3, the FE and analytical models are now overlapping in order to have a complete description on $\mathcal{M}_{X}^{A B}$, i.e. even in the vicinity of the crack tip. This is necessary for the use of the LMG-X-FEM with the remaining coarse $\mathrm{X}-\mathrm{FEM}$ grids. However, these two models are still matched on the boundary $\Gamma_{W}$ alone, in order to make the solution on $\mathcal{M}_{X}^{A} \cup \mathcal{M}_{W}$ be exactly equal to that of the non-overlapping HAXFEM presented in Section 3. This solver has also to yield an approximate X-FEM description defined under the patch, in the overlapped region $\mathcal{M}_{X}^{B}$.

HAX-FEM formulation (eq. 10) is split into the following two coupled problems defined on the part $\mathcal{M}_{X}^{A}$ of the coarse mesh and on the analytical patch $\mathcal{M}_{W}$, respectively:

$$
\begin{aligned}
\mathbf{K}_{X}^{A} \mathbf{U}_{X}^{A} & =\mathbf{F}_{d}-\mathbf{C}_{X}^{T} \Lambda \\
{\left[\begin{array}{cc}
\mathbf{K}_{W} & -\mathbf{C}_{W}^{T} \\
-\mathbf{C}_{W} & 0
\end{array}\right]\left[\begin{array}{c}
\mathbf{U}_{W} \\
\boldsymbol{\Lambda}
\end{array}\right] } & =\left[\begin{array}{c}
0 \\
-\mathbf{C}_{X} \mathbf{U}_{X}^{A}
\end{array}\right]
\end{aligned}
$$

In order to involve a problem on the whole domain $\mathcal{M}_{X}^{A B}$, the coarse traction $\mathbf{K}_{X}^{B} \mathbf{U}_{X}^{B}$ is added to each side of (16) which gives:

$$
\mathbf{K}_{X}^{A B} \mathbf{U}_{X}^{A B}=\mathbf{F}_{d}-\mathbf{C}_{X}^{T} \Lambda+\mathbf{K}_{X}^{B} \mathbf{U}_{X}^{B}
$$

indeed, since $\Gamma_{W}$ belongs to both $\mathcal{M}_{X}^{A}$ and $\mathcal{M}_{X}^{B}$, and $\left.\mathbf{U}_{X}^{A}\right|_{\Gamma_{W}}=\left.\mathbf{U}_{X}^{B}\right|_{\Gamma_{W}}$, one has:

$$
\mathbf{K}_{X}^{A} \mathbf{U}_{X}^{A}+\mathbf{K}_{X}^{B} \mathbf{U}_{X}^{B}=\mathbf{K}_{X}^{A B} \mathbf{U}_{X}^{A B} \quad \text { and also } \quad \mathbf{C}_{X} \mathbf{U}_{X}^{A}=\mathbf{C}_{X} \mathbf{U}_{X}^{B}=\mathbf{C}_{X} \mathbf{U}_{X}^{A B}
$$

Associating a residual to problems (17) and (18) allows us to formulate them in an iterative form which is usually done in the multigrid framework. Moreover, one uses the classical multigrid assumption which consists in neglecting the high frequency unknown of the fine mesh $\Delta \mathbf{U}_{W}^{k+1}=\mathbf{U}_{W}^{k+1}-\mathbf{U}_{W}^{k}$ in the coarse problem (18). The corresponding problems are reported in Algorithm 2.

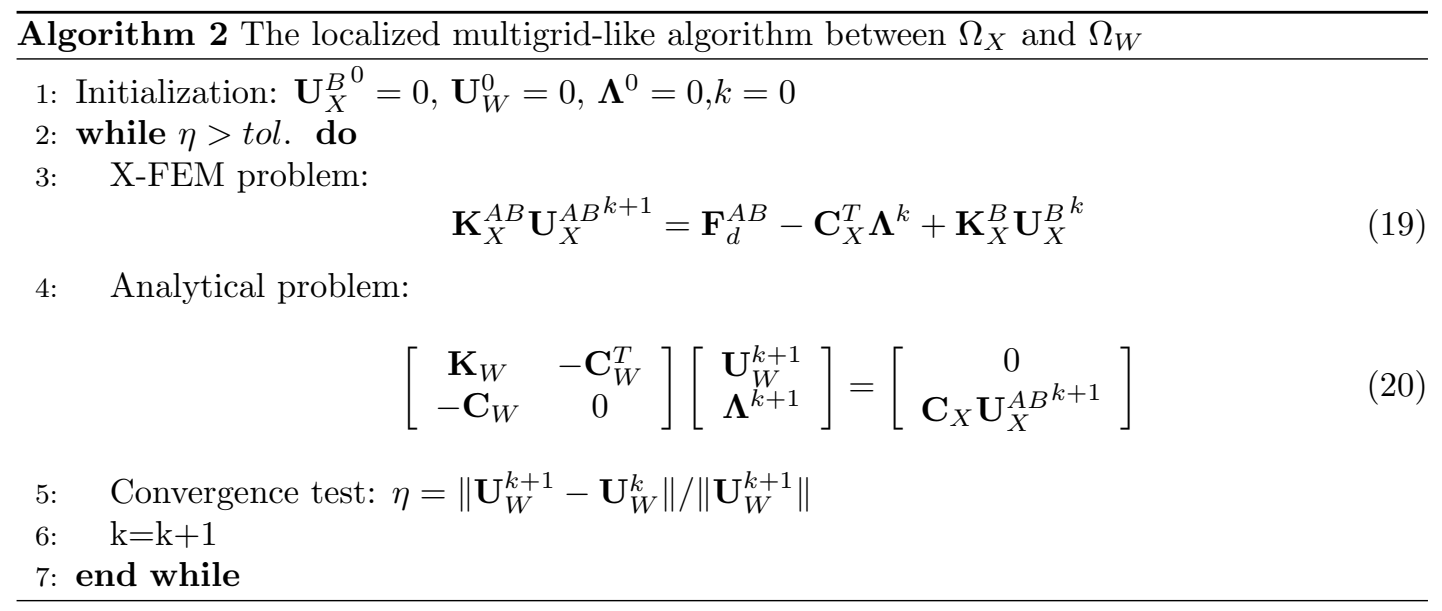

Remark 1. Problem (20) defined on the analytical patch leads to the resolution of a system of fixed and small dimension (usually few tens). As a result, this problem can be solved with 
a direct solver, and no correction stage is required in this algorithm as it was the case for Algorithm 1.

Remark 2. Similarly to [9], this algorithm is a non-intrusive version of the non-overlapping HAX-FEM, since it is entirely based upon standard X-FE computations on the coarse mesh. Indeed, it involves a set of resolutions on $\mathcal{M}_{X}^{A B}$ with an additional loading on the interface $\Gamma_{W}$ only. This step could thus be solved using an existing black-box software in association with a separate piece of code, which may contain the analytical modeling and the intergrids operations.

\subsubsection{Choice of the space of the Lagrange multiplier.}

It is possible, for the space of Lagrange multipliers $\boldsymbol{\lambda}$ to rest on the finite element description which characterizes interface $\Gamma$. In this case, the interpolation reads:

$$
\boldsymbol{\lambda}(x)=\sum_{i \in N_{\Gamma}} \phi_{i}(x) l_{i}^{r}+\sum_{i \in N_{\Gamma} \cap N_{c}} \phi_{i}(x) H(x) l_{i}^{h}
$$

where $N_{\Gamma}$ is the set of nodes of the mesh of $\Gamma$. In $2 \mathrm{D}$, the number of DOF associated with this interpolation of $\boldsymbol{\lambda}$ is equal to $n_{d o f}=2 N_{\Gamma}+2$. Generally, this number is greater than the number of DOF of the analytical model. In such a case, even though this interpolation does not cause any problems with the monoscale formulation, problem (20) becomes ill-posed, because over-constrained. To be solved, this problem has to be reformulated in a least square sense, as follows:

$$
\left[\begin{array}{cc}
\mathbf{K}_{W} & -\mathbf{C}_{W}^{T} \mathbf{C}_{W} \\
-\mathbf{C}_{W}^{T} \mathbf{C}_{W} & 0
\end{array}\right]\left[\begin{array}{c}
\mathbf{U}_{W} \\
\widetilde{\Lambda}
\end{array}\right]=\left[\begin{array}{c}
0 \\
\mathbf{C}_{W}^{T} \mathbf{C}_{X} \mathbf{U}_{X}^{A B}
\end{array}\right]
$$

Used in Alg. 2, this least-square resolution introduces numerical errors that affect the accuracy of the solution. In fact, regarding the size of the space of the analytical solution, this discretization of $\boldsymbol{\lambda}$ is too rich and unadapted.

Another interpolation based on the analytical model is thus proposed to overcome this problem. It consists in the trace on $\Gamma$ of the normal stress fields $\boldsymbol{\Psi} \cdot n$ associated with the displacement modes of the Williams' expansion (2). In order to ensure the equilibrium of $\Omega_{W}$, the three additional functions $\boldsymbol{\Phi}_{I}^{0}, \boldsymbol{\Phi}_{I I}^{0}$ and $\boldsymbol{\Phi}_{I I}^{2}$ are used to force $\boldsymbol{\lambda}$ to be resultant and moment free. If $n_{\Gamma}$ denotes the unit outward normal to $\Gamma$, then the interpolation reads:

$$
\boldsymbol{\lambda}(x)=\sum_{i=I, I I} \sum_{n=1}^{n_{\max }} \boldsymbol{\Psi}_{i}^{n}(x) \cdot n_{\Gamma} l_{i}^{w}+\boldsymbol{\Phi}_{I}^{0} r^{x}+\boldsymbol{\Phi}_{I I}^{0} r^{y}+\boldsymbol{\Phi}_{I I}^{2} m
$$

Note that $\boldsymbol{\Psi}_{I}^{0}, \mathbf{\Psi}_{I I}^{0}$ and $\boldsymbol{\Psi}_{I I}^{2}$ are zero since they correspond to rigid body modes. Therefore, with this interpolation of $\boldsymbol{\lambda}$, the number of DOF is exactly equal to the number of DOF of the analytical model and therefore, problem (20) is well posed. In the sequel, this description will be used since it is more physically sound.

\subsubsection{Analogy with Alg. 1.}

An analogy can be established between Alg. 1 and Alg. 2. On $\Gamma_{W}$, the non-matching interpolations are such that:

$$
\mathbf{C}_{X} \mathbf{U}_{X}=\mathbf{C}_{W} \mathbf{U}_{W}
$$


Therefore, if $\mathbf{C}_{W}$ is square and invertible (if not, a least-square operator should be introduced), a prolongation-like operator $\widetilde{\mathbf{P}}$ can be defined on $\Gamma_{W}$ by:

$$
\mathbf{U}_{W}=\mathbf{C}_{W}^{-1} \mathbf{C}_{X} \mathbf{U}_{X}=\widetilde{\mathbf{P}} \mathbf{U}_{X}
$$

Since the crack faces are traction free, and since the volumic forces are supposed to be zero, then the term $\mathbf{K}_{W} \mathbf{U}_{W}^{k}$ is localized on $\Gamma_{W}$ and is equal to:

$$
\mathbf{K}_{W} \mathbf{U}_{W}^{k}=\mathbf{C}_{W}^{T} \boldsymbol{\Lambda}^{k} \quad \text { and then } \quad \boldsymbol{\Lambda}^{k}=\mathbf{C}_{W}^{-T} \mathbf{K}_{W} \mathbf{U}_{W}^{k}
$$

The term $\mathbf{C}_{X}^{T} \boldsymbol{\Lambda}^{k}$ could then be written differently:

$$
\mathbf{C}_{X}^{T} \mathbf{\Lambda}^{k}=\mathbf{C}_{X}^{T} \mathbf{C}_{W}^{-T} \mathbf{K}_{W} \mathbf{U}_{W}^{k}=\widetilde{\mathbf{P}}^{T} \mathbf{K}_{W} \mathbf{U}_{W}^{k}
$$

and therefore the coarse X-FEM problem of Alg. 2 (19) can be written:

$$
\mathbf{K}_{X}^{A B} \mathbf{U}_{X}^{A B^{k+1}}=\mathbf{F}_{d}^{A B}-\widetilde{\mathbf{P}}^{T} \mathbf{K}_{W} \mathbf{U}_{W}^{k}+\mathbf{K}_{X}^{B} \mathbf{U}_{X}^{B^{k}}
$$

which shows that Alg. 2 and Alg. 1 are written in the same framework.

Therefore, DEK-FEM, which is the combination of these two multigrid techniques, is straightforward and can be seen as a three-scale multigrid algorithm. Even written in the same form, these algorithms are of different nature, and can thus have different convergence rate. The resulting convergence rate of DEK-FEM may be that of the slower. For this reason, a more complex "W"-cycle is considered. The ratio of the number of sub-cycles of type Alg. $2\left(\gamma_{W}\right)$

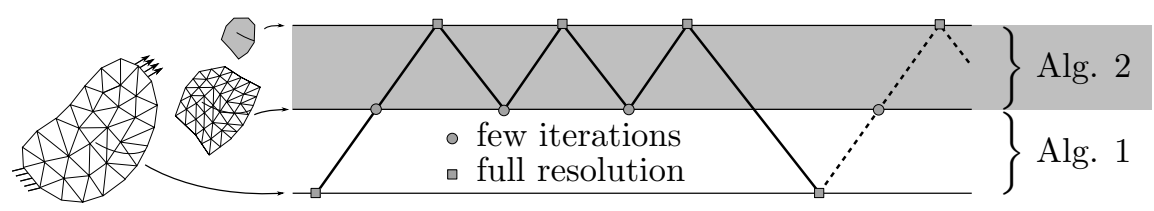

Figure 10: DEK-FEM elementary multigrid cycle parametrized by $\gamma_{W}=3$ and $\gamma_{X}=1$

to that of Alg. $1\left(\gamma_{X}\right)$ can be different from 1 and will be used to balance the convergence rates in order to optimize the efficiency of the overall solver DEK-FEM. An example of the corresponding elementary multigrid "W"-cycle is represented on Figure 10 for $\gamma_{W}=3$ and $\gamma_{X}=1$. The effect of this parameter is examplifyed in the following section.

\section{Numerical example}

In this section, an example already proposed in [20], is used to study the efficiency of $D E K$ FEM. Let us consider the edge-craked plate shown in Figure 11(a), subjected to a uniform shear stress $\tau=1 \mathrm{MPa}$. The plate has a length $L=16 \mathrm{~mm}$, width $w=7 \mathrm{~mm}$ and the length of the crack is $a=3.5 \mathrm{~mm}$. The structure is considered to be in plane strain conditions, with a Poisson's ratio equal to $\nu=0.3$ and Young's modulus $E=100 \mathrm{GPa}$.

Four localized hierarchical meshes made of bilinear Quad4 elements are built. The analytical patch is defined by a set of elements of the finest mesh in the vicinity of the crack tip. The 


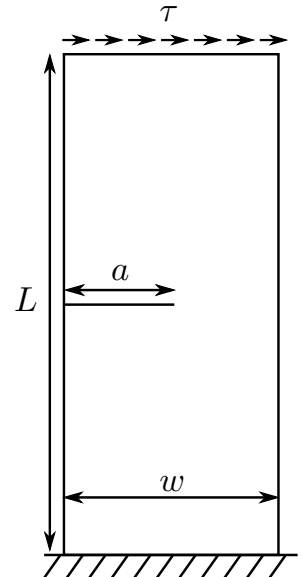

(a) geometry and loading

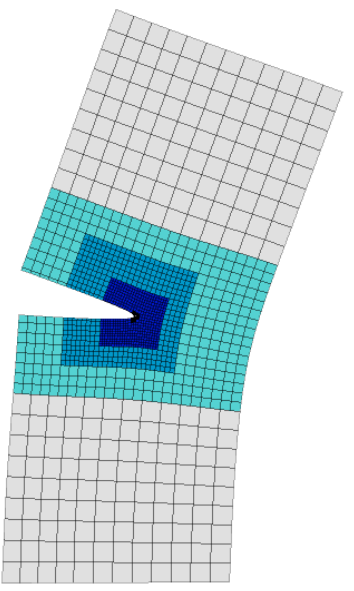

(b) 4 grids and a patch (ampl. fact. $\times 1000)$

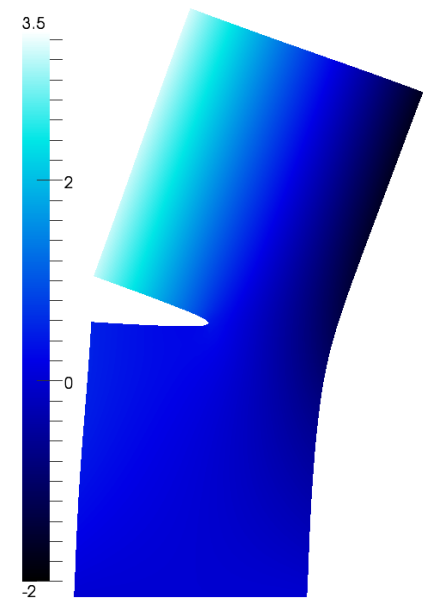

(c) Vertical displacement map in $\mu \mathrm{m}($ ampl. fact. $\times 1000)$

Figure 11: Edge cracked plate under shear

X-FEM meshes, the patch and the crack are plotted on Figure 11(b). Figure 11(c) shows the vertical displacement field in the deformed domain. The plane strain solution of Wilson [36] provides the reference values of the SIF: $K_{I}=34 \mathrm{MPa} \sqrt{\mathrm{m}}$ and $K_{I I}=4.55 \mathrm{MPa} \sqrt{\mathrm{m}}$.

First, the $4 \mathrm{X}$-FEM grids are considered alone to review some properties of LMG-X-FEM. The problem is solved with Alg 1 and the evolution over iterations of the convergence indicator $\eta$ (defined Alg. 1) for each grid is reported on Figure 12(a). One can observe that after only

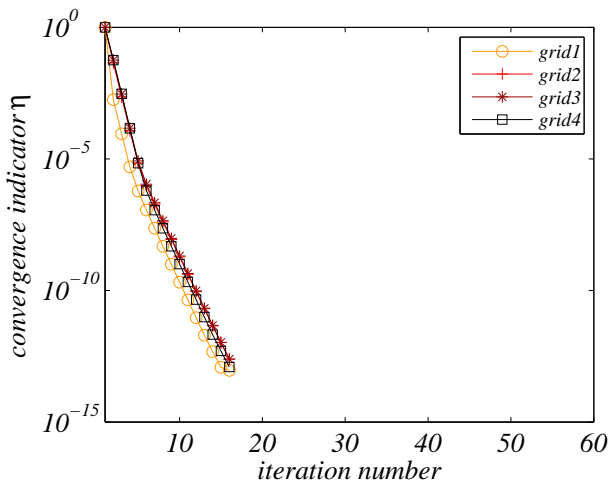

(a) convergence of Alg.1 with 4 grids

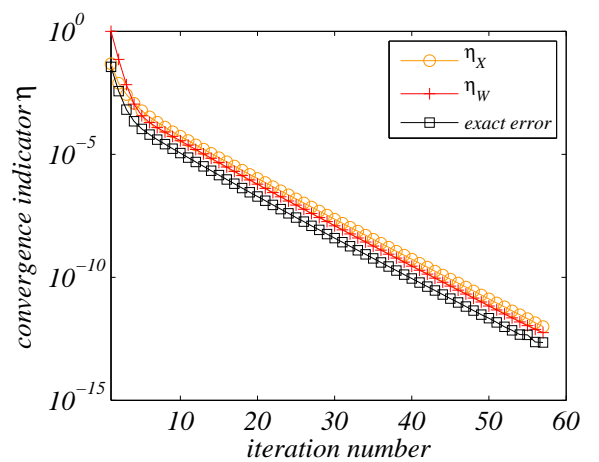

(b) convergence of Alg.2 (1 grid + the patch)

Figure 12: convergence of multigrid Algorithms 1 and 2

15 multigrid iterations, the indicator $\eta$ of the 4 grids are below $10^{-12}$. The fact that the curves of each grid are similar seems to show that all the grids have the same convergence and that 
their indicator $\eta$ are equivalent. Further remarks and properties of this algorithm are given in $[27]$.

Now, Alg. 2 is studied using this example. For this, the coarsest mesh is used alone and the analytical reduced order model is defined in one layer of elements around the crack tip. The problem is successively solved by the direct monoscale method presented in Section 3 whose solution $\left(\mathbf{U}_{X}^{r e f}, \mathbf{U}_{W}^{r e f}\right)$ will stand as a reference, and by the iterative multiscale Algorithm 2. The following exact error indicator can be then introduced to quantify the convergence of the SIF and higher order terms of the Williams' expansion:

$$
\eta_{\text {exact }}=\frac{\left\|\mathbf{U}_{W}^{k}-\mathbf{U}_{W}^{r e f}\right\|}{\left\|\mathbf{U}_{W}^{r e f}\right\|}
$$

This exact measure of the error is plotted over the iterations on Figure 12(b) along with the indicators of the coarse grid $\eta_{X}$ and of the analytical model $\eta_{W}$. One can first observe that the exact error goes to zero, which means that Alg. 2 yields exactly the solutions of the monoscale solution. One can also see that the curves of $\eta_{X}$ and $\eta_{W}$ are similar and also similar to the exact error $\eta_{\text {exact }}$. Therefore, the convergence indicator of each grid can be considered as a reliable error indicator. In the sequel, the indicator $\eta$ of the coarsest grid alone will be used as convergence indicator.

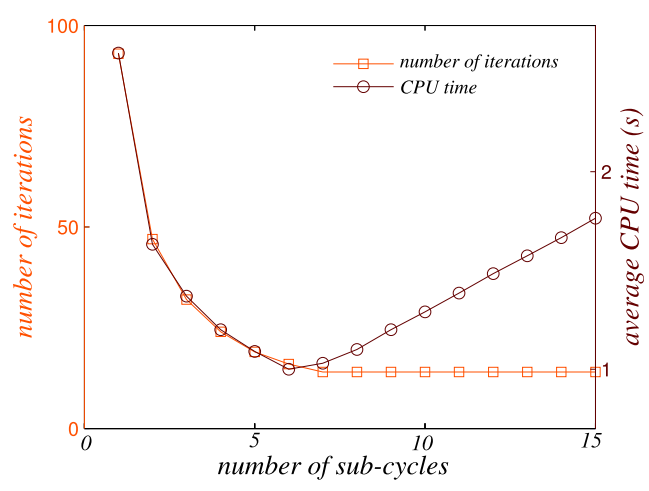

(a) CPU time and number of iterations needed to reach an error of $10^{-12}$ for different values of $r_{\gamma}$

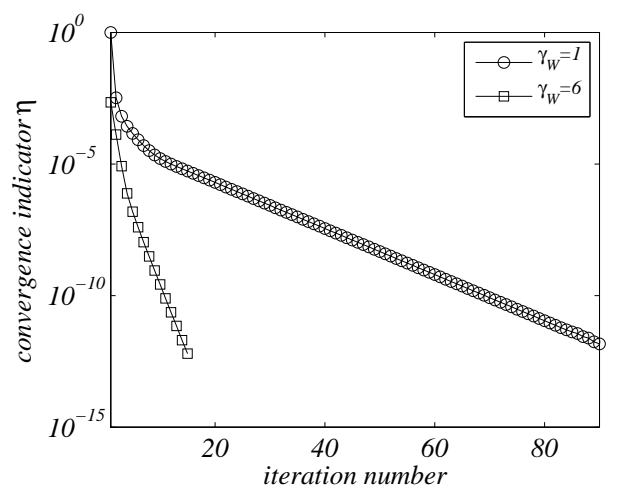

(b) Convergence of $D E K-F E M$ for different values of $r_{\gamma}$

Figure 13: Effect of the ratio $r_{\gamma}$ on the behavior of the algorithm.

A 5-level DEK-FEM (4 X-FEM grids plus the analytical domain) is now used to solve this problem. Even though the convergence of Alg. 2 is linear and quite fast, it is approximately 4 times slower than Alg. 1. Therefore the multigrid solver resulting from the combination of these two algorithms should suffer from this unbalance, if a classical "V"-cycle is used.

In order to highlight this phenomenon, the number of global multigrid iterations needed to reach an error below $10^{-12}$ and the corresponding CPU time are plotted on Figure 13(a) for different values of $r_{\gamma}=\gamma_{W} / \gamma_{X}$. One can observe that when the patch is added at the top of the hierarchy of grids with a naive choice of $r_{\gamma}=1$, an error below $10^{-12}$ is reached within 94 iterations (Fig. 13(b)) whereas it took only 15 iterations without the analytical 
model (Fig. 12(a)). Using a more complex subcycle arrangement $\left(r_{\gamma} \neq 1\right)$, the number of iterations reduces rapidly when the ratio of sub-cycles $r_{\gamma}$ increases. For $r_{\gamma} \geq 6$, it stabilizes at an asymptotic value (16 iterations) for which the convergence rate is equal to that of the LMG-X-FEM. For example, the evolution of the convergence indicator $\eta$ over the iterations is plotted on Figure 13(b) for $r_{\gamma}=1$ and $r_{\gamma}=6$. This sub-cycling requires some resolutions of a very small system on the patch $(12 \times 12$ in this example), so the extra cost is negligible. Thus, for substantially the same computational cost as LMG-X-FEM, DEK-FEM provides, in addition, accurate estimations of the generalized stress intensity factors directly.

Regarding computational cost, the CPU time reduces in the same way and increases again linearly, because the number of sub-cycles increases and the number of global iterations stabilizes. The parameter $r_{\gamma}$ is used to balance the different convergence rates of Alg. 1 and 2. It seems that for this example, an optimal value of $r_{\gamma}$ would be around 6 or 7 for which the resolution time is more than $60 \%$ faster than the naive approach $r_{\gamma}=1$, which represents significant savings. When $r_{\gamma}$ is smaller (resp. greater) than this optimal value, Alg. 2 (resp. Alg. 1) slows down the global algorithm. More generally, since the slope is steeper for the low values of $r_{\gamma}$, one should prefer to use larger numbers of sub-cycles to reduce computational time.

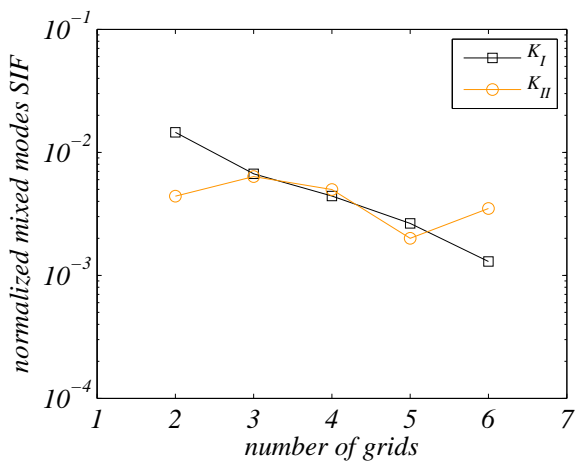

(a) Accuracy of the mixed modes stress intensity factors for different grid numbers

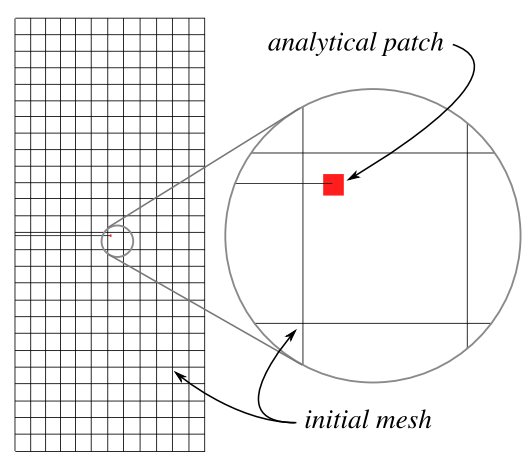

(b) Size of the patch vs size of one element of the initial mesh for 6 grids

Figure 14: Effect of the patch size on the algorithm.

With a fixed coarse mesh, for $r_{W}=3$, and for different grid numbers, Figure 14(a) shows the accuracy of the computed mixed modes SIF. One can observe that the level of accuracy of mode $I$ and $I I$ is similar, and that they are slightly improved when the grid number increases. The size of the analytical patch can therefore be very small without affecting the SIF accuracy. For example, in Figure 14, the size of the patch is divided by 16 when the number of grids goes from 2 to 6 , without the quality of SIF is affected. In this example, we are thus able to consider a patch whose size is 8 times smaller than the size of the element of the initial mesh as shown Figure 14(b) 


\section{Conclusion}

An efficient and accurate concurrent multigrid method for the direct extraction of generalized stress intensity factors is proposed in the present paper. First, a non-overlapping integral matching is employed between a X-FEM model and a localized analytical reduced order model expressed by the Williams' expansion to estimate accurately SIFs with a monoscale method. Secondly, we propose and combine with this method a localized multigrid solver that bridges gradually the scale of the singularity at the crack tip to that of the structure. The global algorithm uses a hierarchy of localized FEM/X-FEM grids, plus an additional analytical model around the crack tip. The localized multigrid method introduced in [27] is used to handle the FEM/X-FEM grids and a multigrid-like algorithm is proposed in a similar manner, to combine the last X-FEM grid and the analytical patch. Despite the ease of use (arbitrary meshes, no overlap, non-intrusiveness... ), three classical tests cases illustrate the ability of the method to provide accurate mixed mode stress intensity factors and also higher order coefficients of the elastic crack tip asymptotic fields directly, as additional degrees of freedom. In addition, some parameters of the method, among which the size of the patch, the space of Lagrange multipliers, the multigrid subcycles arrangement, have been discussed with the support of examples.

The main short-term perspective, concerns the extension of this work to $2 \mathrm{D}$ crack propagation and particularly in the case of non-rectilinear crack (curvilinear cracks, kinks...). HAXFEM was shown to be accurate and efficient to simulate a curvilinear crack propagation in [32], so the extention of DEK-FEM should be straightforward. Two approaches are possible. Either we choose to reduce the size of the patch, and for that, the multigrid will be a major asset, or one can also choose to adapt the analytical modes to take into account the real crack shape. For the first approach, an adaptive refinement method could be developed to generate the optimal hierarchy of grids along with the optimal patch size automatically. Another possible extension of this work is the simulation of dynamic crack propagation. Dynamic stress factors will be extracted in the same way that the static case, up to a multiplicative factor that depends on the crack velocity (see [30]). We will need to define a projection to transfer kinematic fields from patch to the X-FEM grid (and vice-versa) during the propagation.

Apart from the difficulty of the third dimension and aside from the need to introduce the mode III in the analytical model, the extension to 3D is not straightforward. The work done in plane in $2 \mathrm{D}$ will be conducted in each plane orthogonal to the crack front. But the 3D mesh nodes are not aligned in these planes. To manage this difficulty, we can build on the work done by Rannou [28] on level sets handling. The idea is to build, at each step of propagation, a 1D finite element interpolation of the generalized SIF along the crack front. Finally, such a method can also be extended to cohesive cracks or other enhanced model of propagation.

\section{Acknowledgements}

This work is supported by the French National Research Agency (Grant ANR-09-BLAN-000901 RUPXCUBE). The authors would like to thank François Hild and Stephane Roux for fruitful discussions. 


\section{References}

[1] T. Belytschko and J.-H. Song. Coarse-graining of multiscale crack propagation. International Journal for Numerical Methods in Engineering, 81(5):537-563, 2009.

[2] F. Ben Belgacem. The Mortar finite element method with Lagrangian multiplier. $N u$ merische Mathematik, 84(2):173-197, 1999.

[3] H. Ben Dhia and G. Rateau. The Arlequin method as a flexible engineering design tool. International Journal for Numerical Methods in Engineering, 62:1442-1462, 2005.

[4] C. Bernardi, Y. Maday, and A.T. Patera. A new nonconforming approach to domain decomposition: The Mortar element method. In H. Brezis, and J.-L. Lions (Eds.), Collège de France Seminar XI, Pitman, 1994, pages 13-51, 1994.

[5] A. Brandt. Multigrid Techniques: 1984 Guide with applications to fluid dynamics. The Weizmann Institute of Science: Rehovot, Israel, 1984.

[6] H. Bui. Mécanique de la Rupture Fragile. Masson, Paris, France, 1978.

[7] E. Chahine, P. Laborde, and Y. Renard. Crack-tip enrichment in the XFEM method using a cutt-off function. International Journal for Numerical Methods in Engineering, $75(6): 629-646,2008$.

[8] J. Fish and V. Belsky. Multigrid method for periodic heterogeneous media II. multiscale modeling and quality control in multidimensional case. Computer Methods in Applied Mechanics and Engineering, 126(17-38), 1995.

[9] L. Gendre, O. Allix, and P. Gosselet. Non-intrusive and exact global/local techniques for structural problems with local plasticity. Computational Mechanics, 44:233-245, 2009.

[10] S. Ghosh, J. Bai, and P. Raghavan. Concurrent multi-level model for damage evolution in micro structurally debonding composites. Mechanics of Materials, 39(3):241-266, 2007.

[11] A. Gravouil, N. Moës, and T. Belytschko. Non-planar crack growth by the extended finite element and level sets. Part II: Level-Set update. International Journal for Numerical Methods in Engineering, 53(11):2569-2586, 2001.

[12] P.-A. Guidault, O. Allix, L. Champaney, and C. Cornuault. A multiscale extended finite element method for crack propagation. Computer Methods in Applied Mechanics and Engineering, 197(5):381-399, 2008.

[13] O. Jamond and H. Ben Dhia. Propagation of local alterations using Level-Sets within the Arlequin framework. In Actes du 9ème colloque national en calcul des Structues., volume 1, pages $81-86,2009$.

[14] B.L. Karihaloo and Q.Z. Xiao H. Abdalla. Coefficients of the crack tip asymptotic field for wedge splitting specimens. Engineering Fracture Mechanics, 70:2407-2420, 2003.

[15] B.L. Karihaloo and Q.Z. Xiao. Accurate determination of the coefficients of elastic crack tip symptotic field by a hybrid crack element with p-adaptivity. Engineering Fracture Mechanics, 68:1609-1630, 2001. 
[16] P. Kerfriden, O. Allix, and P. Gosselet. A three-scale domain decomposition method for the 3D analysis of debonding in laminates. Computational mechanics, 44(3):343-362, 2009.

[17] D.-J. Kim, J.P. Pereira, and C.A. Duarte. Analysis of three-dimentional fracture mechanics problems: A two-scale approach using coarse-generalized fem meshes. International Journal for Numerical Methods in Engineering, 81:335-365, 2010.

[18] J. Lasry, Y. Renard, and M. Salaün. Extended finite element method for thin cracked plates with Kirchhoff-Loeve theory. Available from http://math.univ-lyon1.fr/ renard/papers/kirchhoff.pdf, submitted, 2010.

[19] G. Legrain, N. Moës, and E. Verron. Robust and direct evaluation of j2 in linear elastic fracture mechanics with the X-FEM. International Journal for Numerical Methods in Engineering, 76:1471-1488, 2008.

[20] X.Y. Liu, Q.Z. Xiao, and B.L. Karihaloo. Xfem for direct evaluation of mixed mode SIFs in homogeneous and bi-materials. International Journal for Numerical Methods in Engineering, 59:1103-1118, 2004.

[21] A.A. Lubrecht and C.H. Venner. Multilevel methods in lubrication. Elsevier, Amsterdam, 2000 .

[22] J.M. Melenk and I. Babuška. The partition of unity finite element method: basic theory and applications. Computer Methods in Applied Mechanics and Engineering, 139:289-314, 1996.

[23] N. Moës, J. Dolbow, and T. Belytschko. A finite element method for crack growth without remeshing. International Journal of Engineering Science, 46:131-150, 1999.

[24] N. Moës, A. Gravouil, and T. Belytschko. Non-planar crack growth by the extended finite element and level sets. Part I: mechanical model. International Journal for Numerical Methods in Engineering, 53(11):2549-2568, 2001.

[25] I.D. Parsons and J.F. Hall. The multigrid method in solid mechanics: part I-algorithm description and behaviour. International Journal for Numerical Methods in Engineering, 29:719-737, 1990.

[26] J.P. Pereira and C.A. Duarte. Extraction of stress intensity factors from generalized finite element solutions. Engineering Analysis with Boundary Elements, 29:397-413, 2005.

[27] J. Rannou, A. Gravouil, and M.-C. Baietto-Dubourg. A local multigrid X-FEM strategy for 3-D crack propagation. International Journal for Numerical Methods in Engineering, 77:581-600, 2008.

[28] J. Rannou, N. Limodin, J. Réthoré, A. Gravouil, W. Ludwig, M.-C. Baetto-Dubourg, J.-Y. Buffire, A. Combescure, F. Hild, and S. Roux. Three dimensional experimental and numerical multiscale analysis of a fatigue crack. Computer Methods in Applied Mechanics and Engineering, doi:10.1016/j.cma.2009.09.013:in press, 2010. 
[29] J. Réthoré, A. Gravouil, and A. Combescure. A stable numerical scheme for the finite element simulation of dynamic crack propagation with remeshing. Computer methods in applied mechanics and engineering, 193:4493 - 4510, 2004.

[30] J. Réthoré, A. Gravouil, and A. Combescure. An energy-conserving scheme for dynamic crack growth using the extended finite element method. Computer methods in applied mechanics and engineering, 63:631-659, 2005.

[31] J. Réthoré, S. Roux, and F. Hild. Hybrid analytical and extended finite element method (HAX-FEM): A new enrichment procedure for cracked solids. International Journal for Numerical Methods in Engineering, 81(3):269-285, 2010.

[32] J. Réthoré, S. Roux, and F. Hild. Mixed-mode crack propagation using a hybrid analytical and extended finite element method. Comptes Rendus de Mécanique, 338:201-126, 2010.

[33] P. Tong, THH Pian, and SJ Lasry. A hybrid element approach to crack problems in plane elasticity. International Journal for Numerical Methods in Engineering, 7(3):297-308, 1973.

[34] G. Ventura. On the elimination of quadrature subcells for discontinuous functions in the extended finite-element method. International Journal for Numerical Methods in Engineering, 66(5):761 - 795, 2005.

[35] M. Williams. On the stress distribution at the base of a stationary crack. ASME Journal of Applied Mechanics, 24:109-114, 1957.

[36] WK. Wilson. Combined mode fracture mechanics. PhD thesis, University of Pittsburgh, 1969.

[37] Q.Z. Xiao and B.L. Karihaloo. Direct evaluation of accurate coefficients of the linear elastic crack tip asymptotic field. Fatigue and Fracture of Engineering Materials and Structures, 25:719-729, 2003.

[38] Q.Z. Xiao and B.L. Karihaloo. An overview of a hybrid crack element and determination of its complete displacement field. Engineering Fracture Mechanics, 74:1107-1117, 2007. 